\title{
Los cambios históricos y la interdisciplina de la ciencia en la innovación pedagógica de la enseñanza geográfica
}

\section{Historical changes and interdisciplinarity of science in pedagogic innovation of the geography teaching}

\author{
José Armando Santiago Rivera \\ Profesor, Universidad de los Andes \\ Táchira, Venezuela \\ Correo electrónico: jasantiar@yahoo.com
}

\begin{abstract}
Resumen: el propósito del artículo es reflexionar sobre la necesidad de innovar la enseñanza de la geografía ante el desarrollo de los cambios históricos contemporáneos, con el apoyo de la interdisciplinariedad. El motivo lo constituye la existencia de una realidad ambiental, geográfica y social que amerita de explicaciones integrales, holísticas, sistémicas y contextualizadas, de tal manera de entender los temas y problemáticas desde la integración de fundamentos teóricos y metodológicos de disciplinas afines. Metodológicamente, se realizó una revisión de bibliografía para estructurar un planteamiento donde se analiza los cambios históricos en la ciencia social durante el siglo XX y la explicación interdisciplinaria para analizar la realidad geográfica. Se concluye al resaltar la importancia de enseñar geografía desde la perspectiva interdisciplinaria, fundada en una visión científica, pedagógica y didáctica, reveladora del origen, cambios y transformación del comportamiento geográfico del objeto de estudio.
\end{abstract}

Palabras Clave: cambios históricos, innovación, enseñanza geográfica, interdisciplina.

Recibido: noviembre 2016

\begin{abstract}
: the purpose of the article is to reflect on the need to develop the teaching of geography an innovative vision supported by interdisciplinarity between geography and history. The reason is the existence of an environmental, geographical and social reality that merits of comprehensive, harmonious and contextualized explanations, so to understand the issues and problems from the integrity of the theoretical and methodological foundations of both disciplines. Methodologically, a review of literature was undertaken to structure an approach where the historical changes in social science is analyzed during the twentieth century and into the interdisciplinary version of the geographic reality. It concludes by highlighting the importance of teaching geography from an interdisciplinary perspective, from a scientific, pedagogical and didactic vision of retrospective accent revealing the causes that have historically caused the geographical behavior of the object
\end{abstract} of study.

Keywords: innovation, geographic education, interdisciplinarity, geography and history.

Aceptado: septiembre 2017 


\section{Introducción}

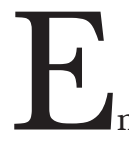

el desenvolvimiento del momento histórico de fines del siglo $\mathrm{XX}$, se manifestaron eventos que permitieron expresar cambios en el rumbo de las tendencias emergentes en los diferentes ámbitos del sistema integral de la sociedad. Por ejemplo, cambió el significado del tiempo, se reveló su aceleramiento; se hizo frecuente reconocer la revolución en la ciencia, la tecnología, la economía y en los medios de comunicación social; es decir, un comportamiento de la época diferente al resto de la evolución histórica, en cuanto al suceder de los acontecimientos y sus repercusiones en la sociedad.

Allí, un viraje destacado fue lo ocurrido en el ámbito paradigmático y epistemológico donde se exhibieron avances trascendentes en lo referido a la consolidación de nuevas versiones epistémicas y, con eso, otras formas de elaborar el conocimiento. Precisamente, ante la importancia asignada a las disciplinas, se evidenció la necesidad de integrar campos del conocimiento como opción para considerar los complejos objetos de estudio, con la relación de fundamentos teóricos y metodológicos de ciencias afines, con el propósito de asumir la explicación del tema y/o problemática geográfica.

En el ámbito educativo, se estimuló la aplicación de la Interdisciplina en la planificación escolar con la exigencia de correlacionar objetivos y contenidos programáticos establecidos en los diseños curriculares, en el tratamiento pedagógico y didáctico de las dificultades ambientales, geográficas y sociales de las comunidades. El resultado fue analizar la realidad desde otras perspectivas facilitadoras del desciframiento de las condiciones existentes en el territorio y en la organización del espacio geográfico, derivado de la combinación disciplinar.

En efecto, lo enunciado dio pie para dar un vuelco a la tradicional transmisividad de contenidos programáticos como la tarea primordial de la enseñanza de la geografía $\mathrm{y}$, al respecto, proponer procesos de enseñanza y de aprendizaje, más ajustados a las circunstancias de la época contemporánea. Como muestra se puede citar la elaboración del conocimiento desde la investigación didáctica, ejercitar la explicación integral y vivencial de los acontecimientos comunitarios cotidianos y diseñar opciones factibles de promover el cambio innovador a la situación estudiada.

De esta forma, al asumir la interdisciplina como iniciativa innovadora en este campo del conocimiento, se tradujo en el mejoramiento de orientaciones sustentadas desde el siglo XIX, como son los casos del determinismo, el enciclopedismo, el pragmatismo pedagógico y la acción didáctica reproductora y memorística. Eso facilitó sostener la actividad pedagógica más vinculada con el entendimiento crítico y constructivo derivado de inmiscuirse en la realidad más allá de lo percibido en forma directa y avanzar hacia las internalidades en procura del conocimiento exhaustivo, sistémico, holográmico y holístico.

Lo expresado determinó metodológicamente realizar una explicación sobre los cambios históricos en las ciencias sociales durante el siglo XX, como parte del proceso 
evolutivo de la cultura occidental. Este suceso revela un conjunto de rasgos donde resaltan las contradicciones, las paradojas y los contrasentidos, como también se desenvuelve con una fisonomía donde resalta la innovación, el cambio vertiginoso y una impresionante creatividad, como base hacia la explicación interdisciplinaria de la realidad geográfica.

\section{Los cambios históricos en las ciencias sociales durante el siglo $\mathbf{X X}$}

Desde fines del siglo XIX hasta los años cuarenta del siglo XX, se desarrollaron condiciones históricas donde resaltó el impulso de la geopolítica y, en especial, los sucesos de la conflagración bélica como rasgos del momento. Se trata de un lapso donde resalta la conflictividad originada por las definiciones territoriales de los EstadosNación, ante los intentos por restaurar los linderos fronterizos originales de las comarcas medievales y/o regiones con identidad geográfica e histórica común.

En ese contexto, en palabras de Grasa (1996), resaltaron las reflexiones sobre los sucesos del momento histórico reveladores del incremento del belicismo y la discordia, con el propósito de evitar el fanatismo ideológico y la irracionalidad del fascismo, aspectos cada vez más en boga en esos tiempos. Fue entonces apremiante diligenciar la armonía, la paz y la concordia ante los excesos compulsivos para originar la conflictividad geopolítica, como de las iniciativas por adueñarse de los territorios originales.

Los eventos calificados como primera y segunda guerra mundial mostraron una panorámica con notables efectos sociales y especialmente, la devastación de los territorios. Las nefastas consecuencias fueron reflejadas en las estadísticas, como en la destrucción de la realidad geográfica. El resultado fueron ciudades arruinadas, campos arrasados, vías de comunicación desmanteladas y la ciudadanía arruinada por las enfermedades, el hambre y el fallecimiento de millones de personas, entre otros aspectos.

Concluida la segunda guerra mundial, se consideró la importancia de asumir como temas significativos la inestabilidad, la conflictividad y la inseguridad mundial. En consecuencia, se impuso el apremio de analizar la inquietante desestabilización geopolítica, además de prestar atención en el posconflicto, al evidente incremento del deterioro ambiental, el desastre en los territorios, la complejidad de la problemática social y la gestión reconstructiva de los países afectados por esta problemática de amplia magnitud planetaria.

Al examinar esta circunstancia histórica, Ferrer (1996), explicó que mientras eso sucedía, en América Latina y el Caribe, existía una complicada realidad sociohistórica donde se resaltaba la coexistencia política del caudillismo militar, los golpes de estado y la autocracia. También la monoproducción agropecuaria y minera extractiva como la base de la estructura económica fuertemente dependiente de los designios de la metrópoli, al igual que actividades impuestas por el colonialismo europeo desde el siglo XV.

El resultado fue la frecuencia de las dictaduras, el despotismo opresor y las tiranías absolutistas. Estos sucesos históricos hicieron posible e imprescindible promover el cambio político desde la confrontación fascista hacia la democracia, la paz y la armonía ciudadana, como base del cambio histórico, con el propósito de garantizar la estabilidad, 
el equilibrio y la concordia. Esencialmente todo apuntó hacia la democrática como el objetivo esencial de la gestión política.

En principio, desde la perspectiva de Hernández (1998), se impuso estimular la permanencia, la seguridad y la regularidad política en clara diferencia a la conflictividad desenvuelta durante el siglo XIX. El fenómeno histórico del caudillismo, la revueltas regionales y los golpes de estado militares ameritaban de la debida atención hacia el fortalecimiento de la condición de Estado-Nación, pues de una u otra forma, originaban el desvió de las ideas políticas de los precursores de la independencia de España y Portugal.

En los hechos ocurridos durante los años cincuenta y sesenta del siglo XX, se comenzó a vislumbrar un escenario histórico donde se descollaron nuevos comportamientos y tendencias en lo fundamental hacia la democracia, la paz y el progreso. Estos aspectos fueron fortalecidos con la creación de organismos de alcance mundial; por ejemplo, la Organización de las Naciones Unidas (ONU) y a nivel regional, la Organización de Estados Americanos (OEA).

En el caso de la OEA, se pretendió poner en práctica una acción renovadora de las condiciones sociohistóricas de los países latinoamericanos y caribeños; en especial, urgía apoyar la democracia como la opción política, un modelo económico con capacidad de transformar el apego a la monoproducción minera y agropecuaria, igualmente un modelo educativo en correspondencia con los desafíos de una sociedad moderna y estimuladora del progreso; es decir, un nuevo modelo de desarrollo integral.

Esta iniciativa asumió el impulso de la ciencia y la tecnología cuyo avance se tornó decisivo en la originalidad y la creatividad en los diferentes ámbitos del conocimiento. $\mathrm{Al}$ analizar esta situación, Moss (1967), consideró la necesidad de tomar en cuenta que: "La ciencia se mueve tan rápidamente que es muy difícil hallarse al día sobre su fantástico y múltiple desarrollo, y ya no digamos entenderlo. Es particularmente dificultoso el prever todas las aplicaciones que puedan surgir de los actuales descubrimientos”(p. 15).

Ya durante los años setenta y ochenta del siglo XX, se hizo común en este continente, percibir acontecimientos donde destacó el progreso científico-tecnológico, la elaboración de propuestas económicas para adecuar el incremento comercial, industrial y financiero a las nuevas condiciones de la época. Aunque asimismo fue destacable el mejoramiento de la calidad mediática de los medios de comunicación social para divulgar los sucesos, los adelantos extraordinarios e inducir la versión del acercamiento mundial más próximo.

El cambio, aunque en el ámbito científico-tecnológico era indiscutible, todavía no tenía la traducción que logró alcanzar a fines del siglo XX. Sin embargo, desde el punto de vista de Núñez Tenorio (1976), en su momento, observó con interés un hecho significativo: Se comenzó a aminorar el tiempo entre la inventiva de la producción en el ámbito de la tecnología y la productividad. La reducción favoreció fortalecer la prosperidad de la industria, además de innovar el mercado con la manipulación de la publicidad.

Indiscutiblemente que un hecho a destacar derivado de este suceso fue la notable innovación de los medios de comunicación social, efectiva en los periódicos, la radio y la televisión. En esta acción se deben resaltar los efectos revolucionarios de los avances científicos y tecnológicos de la industria electrónica y la revolucionaria ingeniería microelectrónica. Con esto fue posible mejorar la calidad informativa, como avanzar su 
divulgación desde los espacios regionales y nacionales hacía la cobertura internacional.

Ya en este momento histórico, gracias al mejoramiento de la acción difusora de los medios, se tuvo la oportunidad de apreciar en forma integral a la realidad de las circunstancias ocurridas diariamente en las diferentes regiones del globo terráqueo. Se trata de la existencia de la situación calificada por McLuhan (1974), como la aldea global. Esta versión concibe la integridad planetaria como un lugar común donde la totalidad mundial está enterada de las situaciones cotidianas ocurridas en el contexto global.

En ese escenario, desde la perspectiva de Naisbitt (1984), se manifestaron los síntomas de una época histórica particular, especifica e individualizada, caracterizada por tendencias y comportamientos donde percibían los cambios con sentido apresurado y aligerado. Por cierto, allí, fue posible percibir en forma rápida como el futuro se hace presente apresuradamente, pues: “...nos hemos movido de lo viejo a lo nuevo. Y continuamos todavía en movimiento, atrapados entre eras y experimentando turbulencia”(p. 9).

Ya en los años noventa del siglo XX, con la divulgación de los acontecimientos de la vida cotidiana, se hizo evidente el sentido y efecto frecuente del cambio apurado y vertiginoso. Además ya estaban en desarrollo los efectos de los acontecimientos históricos de trascendencia, como son los casos del estallido de la Unión Soviética, la fragmentación de Yugoslavia y la caída del Muro de Berlín. Una consecuencia relevante fue el surgimiento del nuevo orden económico mundial, bajo la egida del pensamiento neoliberal.

Conjuntamente, al final del siglo XX, al citado suceso de implicaciones geopolíticas, económicas y mediáticas, se hizo incuestionable gracias a la inventiva y creatividad de la microelectrónica, la posibilidad de divulgar abiertamente las informaciones, las noticias y los conocimientos. Se trata de la realidad comprendida como "la sociedad del conocimiento" y/o la "Explosión del conocimiento". Así, la oportunidad para que cualquier ciudadano, estar en la capacidad de informarse abiertamente sobre los acontecimientos.

En el análisis explicativo de esta posibilidad para la sociedad mundial, Estefanía (1996), colocó en el primer plano la opción del fácil acceso a los datos sobre los sucesos ambientales, geográficos y sociales, como también a la divulgación de los conceptos y prácticas en los diferentes campos del conocimiento; es decir, se abrió una importante oportunidad para elaborar los conocimientos desde diversas perspectivas, en las temáticas y las problemáticas objeto de estudio para los diferentes ámbitos de la ciencia.

En este contexto sociohistórico, surgió una nueva concepción de la verdad de la ciencia. Se trata de la orientación cualitativa. Por cierto, hasta el momento, los fundamentos teóricos y metodológicos del positivismo se consideraban como el sustento indiscutible del conocer valido y confiable, donde el conocimiento se elaboraba como lo seguro, indiscutible y absoluto, derivado de la exactitud, la autenticidad y lo irrefutable.

Sin embargo, pronto fue real la exigencia del viraje en el conocer científico, ante las evidencias de agotamiento y debilidad del positivismo para explicar los acontecimientos inesperados, inciertos, paradójicos y plenos de contrasentidos. ¿Cómo comprender los sucesos de signo impregnados de inseguridad, indecisión y ambigüedad? Con estas indicaciones del momento histórico, inevitablemente se reveló la crisis de la perspectiva científica que superó la permanencia de la especulación metafísica medieval. 
Esencialmente se hizo posible para los criterios establecidos por la ciencia positiva, evitar su condición de incoherentes, poco confiables y escasamente válidos para elaborar la verdad absoluta, cuando en los objetos de estudio, en especial, los temas y problemáticas de la sociedad estaban impregnados de relativismo, confusión y complejidad. Pues, en efecto, el desorden y la confusión ocasionaron dificultades al positivismo para entender la realidad social, cada vez más enrevesada, confusa e indescifrable.

La debilidad positiva se revela en el ámbito paradigmático y epistemológico con los aprietos para dar respuesta explicativa a los eventos impregnados de incongruencias, incoherencias y desatinos. Esto originó evidentemente la desconfianza en lo cierto de origen mecánico y funcional; como también lo inexorable de lo infalible dio paso a la inseguridad, la incertidumbre y al dilema. En consecuencia, la exigencia de otras explicaciones, pues como lo afirmó Lanz (1993):

Los viejos perfiles epistemológicos hacen aguas; los anteriores modelos de análisis están fuera de servicio; las matrices teóricas que legitimaban antes las tomas de partido ya no aportan el fundamento del pensamiento; los antiguos constructos teórico-ideológicos están en decadencia (no sirven ni para postular ni para desear casi nada) Es eso lo que debe entenderse por crisis de paradigmas (p. 12).

El surgimiento de la crisis epistémica ocasionó a fines del siglo XX e inicios del nuevo milenio, un nuevo escenario sociohistórico y como evidencia inobjetable la expresión de una época con una fisonomía desenvuelta en contradicciones y las manifestaciones del progreso, en coexistencia con un ámbito ecológico, ambiental, geográfico y social en franco detrimento; es decir, un rostro complicado, difícil, enredado y confuso, pero también, llamativo, excepcional y sorprendente, caracterizado por:

a) Los hechos posconflicto del segundo evento bélico de alcance mundializado, derivaron en la existencia de un nuevo momento histórico que por cierto ha recibido diferentes denominaciones, tales como globalización, aldea global, nuevo orden económico mundial. Se trata de una época donde el desarrollo científico-tecnológico ha impuesto su sello para justificar históricamente la existencia de condiciones que justifican la existencia de un tiempo con rasgos particulares, individuales y específicos.

b) En estas condiciones sociohistóricas confluyeron un conjunto de cambios donde resaltan la conformación de nuevos paradigma y orientaciones epistemológicas más allá de lo establecido por la ciencia positiva. Fundamentalmente erigió la ciencia cualitativa con planteamientos innovadores para conocer desde la participación activa y protagónica de los investigadores en sus objetos de conocimiento y originar nuevos esbozos científicos al asumir los puntos de vista de quienes viven el tema y/o problemática estudiada.

c) El viraje de la ciencia ha facilitado la posibilidad de investigar los problemas ambientales, geográficos y sociales, desde otras perspectivas, además de los fundamentos y metodologías del positivismo. Quiere decir que en la actualidad, cada tema y/o problema está en la capacidad de ser estudiado desde la orientación científica que es acorde con su propia naturaleza. El resultado ha sido lograr la coherencia entre el objeto de estudio y la estrategia de investigación hacia hallazgos o resultados más ajustados a su desempeño real. 
d) En el ámbito científico está en desarrollo la exigencia de indagar los objetos de estudio desde la perspectiva de integrar dos o más disciplinas para asumir la investigación y obtener un conocimiento integral, exhaustivo y profundo. Por ejemplo, el caso de la historia y la geografía. La primera, acostumbrada a explicar los acontecimientos del pasado y, la segunda, afecta a análisis de la forma cómo los grupos humanos organizan sus territorios y aprovechan sus potencialidades.

e) Las condiciones del mundo contemporáneo también resultan influyentes en mejorar la calidad pedagógica de los procesos formativos de los ciudadanos, pues la complicada realidad merece de ciudadanos cultos, analítico-reflexivos, críticos y creativos. De esta manera se educan las personas con efectos políticos como la demanda la democracia participativa y protagónica. Indiscutiblemente se requieren ciudadanos entendidos sobre su realidad vivida, como también con capacidad de aportar iniciativas de cambio.

f) En el campo de la enseñanza geográfica se hizo imprescindible facilitar la acción pedagógica y didáctica con fines de desarrollar las potencialidades interpretativas de los educandos, desde una visión integral donde se asocien el sentido común, la intuición y la investigación en la calle. Es una acción educativa enriquecedora de la experiencia con la obtención de conocimientos traducidos en la formación de una subjetividad constructiva, con capacidad transformadora de la realidad vivida.

Desde esta perspectiva, se torna interesante superar la postura reduccionista del positivismo exigente de contemplar los acontecimientos, con el propósito de mantener la objetividad del análisis y evitar la formulación de los distorsionantes juicios de valor. Ahora se puede comprender la realidad geográfica, no solo en lo que se aprecia a simple vista, sino también interrogar lo real sobre su pasado, pues se pueden hallar respuestas a las interrogantes emergentes de la observación preliminar del objeto de conocimiento.

Se impone entonces reivindicar los constructos más cercanos y a lo sucedido. Implica abordar el objeto de estudio desde la posibilidad de dimensionar el hecho, en forma concatenada con sus raíces y sus interrelaciones. Es considerar lo visto, pero del mismo modo, examinar su causalidad más allá de lo apreciado, de tal manera de conocer las razones explicativas de su existencia. En efecto, es comprender la globalidad desde la globalidad misma, como producto del proceso histórico.

Desde esta perspectiva se podría suponer como una opción válidamente científica, la oportunidad de vivenciar lo empírico hacia logros contundentes en la transformación de las circunstancias complicadas de las comunidades. Eso traduce la exigencia de dar el salto hacia el tratamiento científico de los problemas ambientales, geográficos y comunitarios de las localidades. Es entonces volver la mirada hacia el lugar en la gestión por mejorar la calidad de vida de la colectividad.

El reto es asumir lo inmediato de lo geográfico como escenario donde los ciudadanos viven su vida diaria, pero también donde piensan, opinan, conversan y emiten sus puntos de vista de una u otra forma, relacionados con su esencia de actores de la geografía vivida. Hoy día es natural apreciar como las personas se pronuncian sobre el mundo vivido y eso es significativo en cuanto es la manifestación de su subjetividad elaborada desde el accionar espontáneo en el ámbito comunitario.

Se trata entonces de ofrecer una explicación más coherente y pertinente de los temas 
y problemáticas de la enseñanza de la geografía generalmente estudiados a partir de la observación-descripción con el énfasis en los aspectos físico-naturales del territorio. Por tanto, al vincular lo geográfico con lo histórico, se puede realizar el esfuerzo investigativo de una forma más integral y avanzar desde las externalidades hacia las internalidades. Así, ante la complejidad de los acontecimientos del mundo contemporáneo, esta visión integral conduce al análisis científico coherente con lo real.

\section{Hacia la versión interdisciplinaria para explicar la realidad geográfica}

Los hechos desenvueltos a fines del siglo XX, comenzaron a revelarse en un escenario de cambios vertiginosos, con el acento complicado y el rasgo de crisis, justificado por la inestabilidad, la incertidumbre y lo imprevisto. De allí el apremio de considerar otras opciones explicativas para poder comprender la nueva realidad sociohistórica; en especial, por ser una circunstancia donde se manifiestan contradicciones relevantes de acento alarmante, aunque del mismo modo, otros interesantes tópicos para la sociedad actual.

Lo complicado emergió como una circunstancia habitual y cotidiana, entre otros aspectos, como consecuencia de la forma como la sociedad obtuvo el acceso a las noticias, las informaciones y los conocimientos. Los medios asignaron una significativa importancia al hecho de facilitar simultánea y al instante, los datos necesarios para informar a la colectividad sobre diversos sucesos ocurridos en diferentes regiones del escenario mundial y con eso poder emitir una versión personal sobre la realidad vivida.

En palabras de Hollman (2008), la realidad se tornó globalizada gracias a la acción mediática, pero del mismo modo se pudo obtener los datos sobre los problemas ambientales, geográficos y sociales de suceder diario. Esto hizo fortalecer la condición de enrevesada, adversa y caótica de la panorámica sociohistórica del mundo contemporáneo. Pronto fue evidente apreciar una circunstancia indicadora de un comportamiento confuso, anárquico y desorganizado; es decir, el globo terráqueo en su existencia concreta.

Además de la ruptura de la estabilidad histórica tradicional, se revela en forma cierta e indiscutible, con la impresionante revelación del desarrollo del movimiento acelerado como característica de la época. Aunque el sorprendente ritmo del progreso de la ciencia y la tecnología, también han originado la sensación del rápido cambio ante la forma tan violenta como la producción sustituye los dispositivos creados con el signo de su rápida obsolescencia.

En el contexto de la época actual, el sentido del cambio se muestra entonces como una característica del momento presente claramente apreciado en el extraordinario impulso económico y financiero; por ejemplo, en cada día se pueden obtener con facilidad, datos sobre producción, mercado, comportamiento de las bolsas y la conducta de los precios de las materias primas. Asimismo, las dificultades económicas de las colectividades en diversas regiones del planeta son divulgadas cotidianamente por la acción mediática.

En consecuencia, en el criterio de Gurevich (2005), la época, además de los casos citados, igualmente revela características, tales como: la actualidad de las tendencias hegemónicas en conflicto durante el siglo XX, los cambios geopolíticos, las tendencias homogeneizadoras del pensamiento único, la presencia de inquietantes contradicciones, 
la discordia cotidiana y el incremento de los niveles de pobreza crítica, entre otros aspectos. Así, los impresionantes adelantos y los desaciertos conforman un panorama histórico inquietante derivado de las contradicciones, incoherencias y desacuerdos.

El resultado lo constituye un suceso desenvueltos en un ambiente de conflictividad, negociaciones y transacciones de diversa índole. Aunque sin lugar a dudas en ese ámbito globalizado es lo económico y lo financiero los aspectos más resaltantes, pues han logrado el alcance mundial en su propósito de crear un mercado bajo la egida del modelo neoliberal, el incentivo de la competitividad y el control de los recursos naturales a través de las empresas multinacionales, complementado por alianzas, tratados y acuerdos donde predomina lo comercial y el flujo de capitales.

Por tanto, en la opinión de Kay (2001), la ciencia geográfica se encuentra ante el desafío de explicar el momento histórico, pues urge advertir la distancia entre la exagerada acumulación de capital y la merma de la calidad de vida de los ciudadanos; en especial, el deterioro de la salud y el bienestar de los ciudadanos del mundo globalizado. Allí, un reto es descifrar y contrarrestar el efecto neoliberal que impregna al contexto sociohistórico actual y revelar sus efectos en la sociedad.

Entre las opciones de la ciencia geográfica para abordar la enrevesada realidad histórica, se ha promovido la interdisciplinariedad, como es el caso entre la historia y la geografía, para citar un ejemplo. Se trata de un acto conducente a ejercitar el acto de descifrar el comportamiento sociohistórico de la época. Allí, lo prioritario será develar las situaciones estructuradas por el poder dominante al intervenir en el uso del territorio. Por tanto, se trata de una excelente posibilidad analítica para mostrar cómo el capital aprovecha lo natural y organiza el espacio geográfico desde su perspectiva neoliberal.

En una reflexión sobre este acontecimiento, opinó Ríos (2004), que se torna imprescindible considerar la contextualización del objeto de estudio en su escenario de su época. Con eso se pretende comprender el sentido de complejidad y de cambios acelerados de las circunstancias cotidianas perfilantes del presente momento de acento enrevesado. Eso implica apreciar los sucesos en su dimensión temporo-espacial y es su rápida transformación.

Lo enunciado supone, reflexionar sobre la viabilidad de la explicación en el propio acontecimiento, desde una visión analítico-interpretativa del aprovechamiento del territorio y la organización del espacio geográfico. Es contactar lo real entendido en el marco de los contratiempos vividos por la sociedad e involucra la obligación de ventilar opciones de cambio; especialmente, entender el mundo vivido en su explicación presente-pasado, desde las perspectivas de la colectividad que lo habita.

En efecto, eso requiere opciones innovadoras analíticas coherentes con lo ocurrido ante la exigencia de apreciar los objetos de estudio geográficos desde lo interdisciplinario en el contexto histórico donde se produce. Eso responde de acuerdo con Martínez (2004), a la necesidad de asumir la realidad desde una fundamentación científica más allá de la visión parcelada positiva. Por tanto, ante la complejidad de la época, la visión fragmentada-descriptiva-determinista, es razonada como un obstáculo epistemológico.

Desde este punto de vista, ante las nuevas condiciones históricas, urge optar por otra alternativa científica que asigne relevante importancia a la visión de totalidad y conjunto. En el presente momento histórico, la realidad no puede ser explicada solamente desde 
sus partes constitutivas, sino como una situación total, comportada como dinámica y en procesos de cambios permanentes. Significa que la atomización, la linealidad y el mecanicismo, deben dar paso a procesos ecológicos, holísticos y sistemáticos.

En la opinión de Rodríguez (2008), la fragmentación y el determinismo han representado para la geografía desvirtuar la esencia de la explicación de la realidad. El afecto al determinismo y a la descripción geográfica, tan solo ha servido para detallar y enumerar a manera de inventario, los rasgos físico-naturales y humanos del paisaje. Actualmente, eso conduce a una falsa neutralidad y el apoliticismo sustentados en subyacentes mecanismos del poder para preservar el atraso y el rezago de los pueblos.

Hoy día, la interdependencia e interrelaciones obligan a miradas de conjunto. Lo enrevesado de las situaciones exige ser comprendidas en su complejidad y cambio vertiginoso como totalidades. Este cambio paradigmático trajo consigo la obligación de revisar los planteamientos científicos ante la debilidad originada por la dificultad para continuar con análisis a través de los fragmentos constitutivos de las circunstancias ambientales, geográficas y sociales.

Al respecto, en su momento Tovar López (1983), afirmó: “...en efecto es objeto de la Geografía 'aportar una explicación de la organización diferenciada (sitios, lugares, regiones) del espacio estructurado por los grupos humanos (sociedad, nación, pueblo, comunidad) dentro de condiciones históricas dadas" (p. 12). Por las razones expresadas, la finalidad científica del enfoque obedece a la posibilidad de desdibujar la ideología dominante organizadora del comportamiento de la dinámica del espacio geográfico.

De acuerdo con Aponte (2006), este integración científica asume como argumento esencial a la oportunidad de abordar la dinámica, complejidad, heterogeneidad y especificidad del espacio geográfico. La opción apunta a apreciar integralmente la armonía del tiempo histórico y el espacio geográfico. Eso admitirá la explicación sustentada en la reflexión interpretativa de la evolución del pasado y lo actual, para concebir cómo los grupos humanos han construido su realidad geográfica.

Desde este punto de vista, se trata de develar la estructura sustentadora de la realidad geográfica, bajo el designio del grupo social que ejerce el poder controlador del suceder de los acontecimientos del espacio. El privilegio del grupo dominante se plasma evidentemente en los constructos que emergen como realizaciones ciertas representativas de la evolución histórica del espacio geográfico. Por eso la geohistoria revela las formas como la ideología ha organizado y organiza su entorno en condiciones históricas dadas.

En palabras de Santaella (2005), el evidente sesgo y trasfondo político e ideológico significa asegurar la alienación explicativa y evitar la reflexión critico-interpretativa de los hechos históricos. En efecto, las mismas circunstancias del mundo globalizado han facilitado el incremento de nuevas posturas analíticas para descifrar los sucesos desde una perspectiva más vinculada con la dinámica social, al valorar las temáticas y problemáticas asociadas con la complejidad del momento y la vida cotidiana.

Con la ciencia geográfica ocurre una desestabilización de su orientación científica, debido a la formulación de teorías que abordan de manera particular los objetos de conocimiento de la realidad ambiental, geográfica y social. Indiscutiblemente ahora se da especial atención a las dificultades de acento apremiante para la sociedad; por ejemplo, el calentamiento global, los desastres socio-naturales, el hacinamiento urbano, 
el deterioro ambiental, los conflictos bélicos, la xenofobia, la movilidad sur - norte, entre otros.

A lo expuesto, según la opinión de Araya (2004), las adversas circunstancias requieren de una nueva conciencia ambientalista orientada hacia la protección de las condiciones óptimas de los territorios, como preservar los equilibrios ecológicos y con ello, mejores condiciones de vida para la sociedad. Asimismo, se reclama una Educación Geográfica que sensibilice a los grupos humanos sobre la imperiosa necesidad de una convivencia entre la sociedad y la naturaleza coherente para optimizar la calidad ecológica, ambiental y social

La dificultad que confronta la ciencia geográfica obedece al planteamiento de diversas corrientes explicativas, donde todavía los fundamentos teóricos y metodológicos más aplicados para elaborar el conocimiento geográfico, son los de naturaleza descriptiva. De acuerdo con Villanueva (2002), con la geografía descriptiva dedicada a enumerar, detallar, particularizar, individualizar y pormenorizar las características geográficas del objeto de estudio dificulta entender lo real, pues se trata de solo determinar sus partes y/o fragmentos.

Desde esta perspectiva, la geografía ha comenzado a encontrar otros problemas, además de los tradicionales, con el resultado de complejizar su labor científica. Por tanto, es indudable que sus contratiempos están relacionados con las repercusiones de la crisis paradigmática; en especial, en la forma de abordar los problemas geográficos. En el escenario de los cambios dinámicos y enrevesados, hay dificultades para explicar con la mera descripción, las dinámicas territoriales y espaciales del mundo contemporáneo.

Ya desde los años ochenta del siglo XX, en la opinión de García Ballesteros (1986): "La Geografía, en tanto que ciencia social, ha experimentado profundas transformaciones en los últimos decenios, en gran parte motivadas por la incapacidad de los enfoques tradicionales para hacer frente a las nuevas necesidades sociales (...)”(p. IX). En los años noventa, las críticas abundaron sobre la vigencia de los fundamentos descriptivos y deterministas de la geografía, poco coherentes con las realidades geográficas actuales.

La explicación de la realidad geográfica, desde el positivismo, tiene hoy día apuros debido a su apego a la forma fragmentada y parcializada, como se asume los objetos de conocimiento. Además, el cambio paradigmático donde la ciencia cualitativa se ha convertido en una opción válida para conocer, exige la apreciación integral de sus acontecimientos en su existencia real y concreta. En tal sentido, para responder a esta época, de acuerdo con Gurevich (1994), la geografía como ciencia debe tener como:

...objetivo analizar, interpretar y pensar críticamente el mundo social. Por ello, le cabe a nuestra ciencia la tarea de comprender cómo se articulan históricamente la naturaleza y la sociedad, pues las distintas formas de organización espacial son el resultado del particular modo en que las sociedades en determinados momentos históricos se relacionan con la naturaleza, transformándola según sus necesidades e intereses (p. 71).

En función de lo enunciado, estudiar las circunstancias ambientales, geográficas y sociales del contexto global, traduce en la necesidad de proponer remozadas visiones científicas con capacidad para descifrar la complejidad de los hechos, derivados de la relación entre la sociedad y la naturaleza. No es el caso de contemplar la realidad y 
buscar la opción metodológica acorde con la objetividad solicitada por el positivismo. Ahora la vía de la ciencia se aboca a apreciar la realidad, pero también interrogar lo sucedido.

En la opinión de Santaella (2005), la ciencia geográfica tiene, en efecto, la opción para tratar sus temas de interés, pero también la necesidad formularse preguntas de la misma sociedad sobre su vida pasada y actual. Esto origina desarrollar la integración; por ejemplo, de la geografía y la historia, con el propósito de analizar los objetos de estudio desde una perspectiva que vincula a ambas disciplinas. Significa ver al presente con una visión integral de las disciplinas para estudiar su coexistencia, en lo actual, el pasado y el futuro.

La visión explicativa del mundo actual tiene en la ciencia interdisciplinaria una excelente oportunidad para ofrecer planteamientos científicos coherentes con los desafíos de comprender el mundo vivido de manera más analítica y crítica. La armonía de los fundamentos teóricos y metodológicos de ambas disciplinas, implica vislumbrar el análisis desde el presente hacia el pasado, como viceversa; así, entender la forma cómo los grupos humanos utilizan su territorio y organizan su espacio geográfico.

Al estudiar esta situación, Santarelli y Campos (2002), comentaron que se trata de una opción orientada a ofrecer una visión reconstructiva de los hechos, con un proceso de intervención directa del territorio en condiciones históricas. El propósito es indagar los cambios de época en periodos claramente definidos, como mostrar las situaciones originadas por los mecanismos de dominación ideológica y política del momento. De esta forma, se visibiliza la realidad en sus expresiones históricas, como también geográficas.

Por consiguiente se torna indispensable asumir la interdisciplinariedad. Eso supone apropiarse las situaciones de la realidad en su condición de escenarios complejos, dinámicos y en transformación permanente donde las partes se diluyen en el comportamiento de los acontecimientos. Es considerar la realidad como totalidad percibida en su desempeño cotidiano donde las circunstancias tienen el sentido y significado comunitario como escenario de la vivencia de lo inmediato.

Eso implica integrar conocimientos y prácticas de las disciplinas afines al objeto de estudio. De allí el propósito fundamental de indagar su existencia e interpretar el momento histórico en que ocurre en la gestión por develar la ideología dominante, en sus actos y realizaciones. Ahora es esencial orientar la indagación para obtener la forma cómo la sociedad organiza el espacio geográfico al utilizar las potencialidades del territorio desde los argumentos establecidos por las fuerzas de poder.

Esto conlleva detectar el comportamiento de las fuerzas políticas, económicas y sociales expresadas en el espacio construidas. Se trata entonces de buscar respuesta a interrogantes, tales como las siguientes: ¿Cómo se ha construido la situación geográfica comunitaria?, ¿Qué función ha desempeñado la clase dominante en la organización del espacio?, ¿Cómo se revela en el espacio la fuerza constructiva de la clase dominante?, ¿Cuál será la tendencia histórica del comportamiento de la clase dominante?

El análisis, en función de las interrogantes planteadas, tiene que ser complementado, yendo a la búsqueda de las internalidades históricas de lo que sucede. Es no limitarse a contemplar las externalidades de los hechos con la simple descripción de lo que acontece, sino ir en procura de los fundamentos convertidos en argumentos que dan 
razón a lo que se presenta. Es imprescindible que los estudios históricos deban abocarse a interrogar los hechos para descifrar las internalidades que subyacen en lo que se observa a simple vista.

De acuerdo con este enfoque, la visión histórica facilitará a la iniciativa geográfica estar en capacidad de descubrir o redescubrir la estructura predominante del momento. El propósito es encontrar los indicios que ayuden a establecer los conceptos emergentes hacia la conformación de las categorías reveladoras de la realidad de lo existente. Es encontrar lo probatorio de los argumentos explicativos de la situación geográfica, derivados de interpretar lo visible y esculcar en los actores comunitarios las fuerzas constructivas.

Precisamente, se impone realizar el interrogatorio en la gestión por encontrar respuesta a las siguientes preguntas: ¿Cómo era antes la situación?, ¿Cómo ha evolucionado?, ¿Cómo se desarrollan las características que definen la época que se vive?, ¿Cómo están organizadas y cómo funcionan?, ¿Cuál es la realidad geográfica y sus problemas socioeconómicos?, ¿Por qué se dan esas dificultades en el colectivo social?, ¿Hacia dónde se orientan las tendencias y el comportamiento de los tiempos?, entre otras interrogantes.

En consecuencia, la enseñanza geográfica para adecuarse al cambio paradigmático debería dejar a un lado el desarrollo los procesos de enseñanza y de aprendizaje en forma transmisiva y parcelada, a la vez que abocarse a suscitar cambios explicativos, entre los cuales vale citar el estudio de situaciones de la vida diaria. Es decir, centrarse en el tratamiento pedagógico y didáctico de los problemas de la comunidad, de la vida diaria, del entorno inmediato. Allí la interdisciplinariedad se práctica en el hecho de asumir lo real, tal como es en su condición de totalidad vivida en su dinámica y permanente transformación.

Se impone entonces promover una visión integral para reflexionar sobre la realidad geográfica comunitaria como el objeto de estudio, con fines científico, pedagógicos y didácticos. Esta iniciativa encuentra en la versión interdisciplinaria de la ciencia, a una excelente oportunidad para ajustar los fundamentos teóricos y metodológicos en una unicidad epistémica constructiva en forma acertada y coherente con la geografía vivida en los ámbitos de lo cercano e inmediato.

En la complejidad del mundo contemporáneo, se impone la necesidad de romper con el acento disciplinario tradicional, pues no todos los objetos de estudio científico se pueden analizar desde la perspectiva de una sola ciencia. Lo enredado de los problemas trae como exigencia acercarse a otras disciplinas con el propósito de vislumbrar otras opciones analítico-criticas facilitadoras de otras experiencias y conocimientos más contundentes en el momento de descifrar las situaciones asumidas.

Lo indicado es básico para propender cambios e innovaciones a la enseñanza de la geografía limitada a transmitir contenidos programáticos. Ahora se abre la posibilidad para asociar las nociones y los conceptos gracias a la correlación interdisciplinaria que facilite estudiar no solo los contenidos, sino también asumir temas y problemáticas en forma situacional contextualizadas en el ámbito comunitario donde ocurren. Es vivenciar la interdisciplinariedad en la explicación de la realidad comunitaria inmediata.

¿Hacia dónde va la innovación en la enseñanza de la geografía fundamentada en la interdisciplinariedad? Dar respuesta a esta interrogante supone razonar sobre 
el desarrollo curricular de las asignaturas geográficas, como la necesidad de formar integralmente a los ciudadanos, de tal manera que sean capaces de entender su realidad ambiental, geográfica y social vivida. Por tanto, innovar significa atender a los siguientes aspectos:

a) El énfasis disciplinar geográfico determinista. El hecho de preservar esta orientación decimonónica, resta méritos a la enseñanza de la geografía valor formativo para ser una opción válida en la formación de los ciudadanos que viven la complejidad del mundo globalizado. La innovación sería conveniente tan solo al colocar al determinismo en el marco de la coherencia y pertinencia de sus fundamentos y evitar preservar su tradicional exclusividad en la explicación geográfica.

La geografía tiene en opción interdisciplinaria la posibilidad para explicar las situaciones geográficas acorde y ajustada a las circunstancias de la época; es decir, puede recurrir a su condición de ciencia integradora de conocimientos facilitadora del desarrollo de investigaciones con notable éxito científico y pedagógico. Pero el hecho de vincularse con otras disciplinas, enseñar geografía tiene la posibilidad de mejorar su calidad formativa; por ejemplo, con la historia, la política, la economía y la sociología.

b) El acento absoluto, categórico, definitivo e inmutable del conocimiento obtenido con la actividad hipotético-deductiva, puesta en práctica para conocer la realidad. Lo preocupante de este rasgo es que el conocimiento obtenido es inobjetable, indudable, incuestionable e irrefutable. Se trata de un constructo derivado de un marco teórico garante de la verdad aportada por la ciencia geográfica al estudiar una realidad, cuya experiencia se concibe que pueda ser repetida y derivar en los mismos resultados.

Por tanto, innovar la enseñanza de la geografía implica romper con el sentido y significado de absoluto que el positivismo ha exigido al conocimiento verdadero. En consecuencia, dar el salto hacia el relativismo traduce la posibilidad de estudiar la realidad desde la perspectiva de lo falible, lo dinámico, lo vulgar y lo cotidiano, pues allí la investigación puede ser realizada en forma espiral, reconstructiva y retrospectiva. En lo relativo, enseñar geografía es aproximarse a lo real en su vivencia cotidiana en lo que es, sin las ataduras positivas, para construir una verdad de implicaciones sociales transformadoras y liberadoras, derivada de la opinión de sus propios actores.

c) La dispersión de los rasgos de lo físico-natural del territorio, tiene como intención evitar la comprensión analítica, reflexiva y crítica del aprovechamiento de las potencialidades de lo natural por el capital y desviar el conocer de su dinámica espacial. Es orientar la descripción de la realidad a partir del estudio del detalle como porción aislada, particularizada y desfasada de lo real, para concebir luego, con la sumatoria, una visión sumativa del territorio. Es estimar la situación como acumulación de datos.

Con la interdisciplinariedad, enseñar geografía evita la dispersión de la realidad, pues es posible aproximarse a ella en sus vivencias donde proliferan los puntos de vista de los ciudadanos y los hechos se desenvuelven con naturalidad. Por tanto, lo real es existencia concreta del cambio habitual donde confluyen sus diversos aspectos sin fronteras de ningún tipo, ni diferencia, sino de realidades concretas en dinámico cambio. Este aspecto es esencial en los cambios aspirados por la innovación de la enseñanza de la geografía. .

d) La preservación de la objetividad científica por el hecho de reproducir con exactitud la circunstancia observada con el propósito de evitar la manifestación del 
criterio personal que rompa con la neutralidad, el apolitismo y la desideologización requerido para concebir la verdad científica. Así, lo real es copiar la situación tal y como ella acontece, pues lo objetivo debe ser ecuánime e imparcial. Cualquier revelación de subjetividad merma la cientificidad que debe poseer.

Gracias a los cambios emergentes en los paradigmas y en la epistemología cualitativa, innovar en la enseñanza de la geografía puede ocurrir al estimar una concepción de la objetividad más ajustada a las condiciones de la época y del sucesos investigado. Hoy día es posible mejorar la apreciación de lo objetivo, donde tiene mucho que ver la subjetividad de los ciudadanos actores de los objetos de estudio; es decir, es otra verdad más de acento relativo concebida desde los criterios de las personas sobre su realidad vivida. Eso permitirá a la enseñanza geográfica ir al entorno inmediato en procura de los puntos de vista de los habitantes comunitarios y conocer sus impresiones sobre el contenido geográfico escolar.

e) La explicación objetiva debe estar apegada al mecanicismo, la linealidad y la funcionalidad que caracteriza a los procesos de la naturaleza y como tal sirve para comprender los acontecimientos sociales. De allí que los fundamentos citados son referentes obligados en el análisis de cualquier tema y/o problemática geográfica; en otras palabras, se debe aplicar un método cuyos pasos deben ser planificados en forma estricta y rígida, como lo demanda la ciencia positiva.

Este afecto tradicional permite a la enseñanza de la geografía acoger a las orientaciones del paradigma cualitativo de la ciencia, con el propósito de indagar la realidad comunitaria desde procesos abiertos, flexibles y de actividades reacomodables. Es la oportunidad para innovar su labor formativa al poner en práctica los fundamentos teóricos y metodológicos de la fenomenología, la etnografía y la investigación acción en sus labores pedagógicas y didácticas, de tal manera de encontrar datos en la subjetividad de los ciudadanos.

f) La descripción como labor enumerativa, inventario, catalogo, compilación y clasificación de los rasgos característicos del territorio. Por tanto, entender la realidad geográfica trae como consecuencia proceder a detallar los atributos que posee, en forma puntualizada. Ellos son dados a conocer la narración de lo observado en los aspectos pormenorizados constitutivos del objeto de estudio.

La enseñanza de la geografía da el salto innovador al avanzar desde la descripción enumerativa de los rasgos del territorio hacia la visión interdisciplinaria que sustenta el desarrollo de la investigación de los temas y problemáticas geográficas diagnosticados en la comunidad. Eso supone ver lo real en su existencia vivencial cotidiana donde los sucesos ocurren en el escenario de lo natural y lo espontáneo, concebido como totalidad sistemática.

g) La tarea básica y esencial de la enseñanza de la geografía como disciplina es transmitir los contenidos programáticos. Así, la formación de los ciudadanos se limita a acumular datos referidos a características de la realidad de los territorios, como extraviar y despistar la explicación analítico-critica de la realidad social. El énfasis transmisivo pretende reproducir lo real en forma rigurosa, inflexible e implacable, pues es imprescindible preservar la objetividad garante de lo científico.

La apreciación innovadora se revelará al asignar la prioridad a la elaboración del conocimiento con el apoyo de la investigación didáctica. En efecto, es estimular la 
acción indagadora como la tarea cotidiana del aula de clase. Eso significa estudiar la teoría para luego aplicar los conocimientos en la práctica, como también desde la práctica construir una nueva teoría. En otras palabras, crear conocimientos con efectos significativos en la conformación de la subjetividad personal argumentada y cuestionadora.

h) Los procesos pedagógicos y didácticos deben estar orientados a facilitar la reproducción de los contenidos programáticos, tal y como están establecidos por el ente oficial. Es preciso conservar la naturaleza de lo indicado por el programa; en especial, seguir la secuencia establecida sin modificar su estructura y menos desviar la dirección de la enseñanza y el aprendizaje hacia otros tópicos relacionados con la realidad.

Aquí la innovación en la enseñanza geográfica obedecerá a utilizar el contenido programático como base teórica para fundamentar la aplicación en el estudio de situaciones afines detectadas en el ámbito comunitario. Por tanto, es apremiante tener claridad en la teoría geográfica para poder comprender la complicada realidad en estudio. Se trata de superar el intelectualismo tradicional por una intelectualidad también operativa, dialéctica y constructiva capaz de confrontar dialécticamente la realidad geográfica.

i) La memorización como la manifestación de lo aprendido. Es, en consecuencia, la tarea a cumplir por el docente en el proceso de enseñanza y de aprendizaje de la geografía. La obligatoria tarea es enseñar el contenido libresco con la clase magistral, el dictado, el dibujo, la copia y el calcado, pues facilitan reproducir con alto nivel de fidelidad el conocimiento absoluto enunciado para ser transmitido en el aula de clase.

En este aspecto innovar significa superar la memorización por el ejercicio cotidiano frecuente de la reflexión y el análisis acucioso donde lo fundamental sea el agite del pensamiento razonado, cuestionador, dialéctico y constructivo. En el mundo actual donde fluyen las noticias, las informaciones y los conocimientos en forma abierta y sin limitaciones, además de la vivencia en la localidad de la complicación ambiental, geográfica y social, urge activar la mente con razonamientos agiles y críticos.

Los acontecimientos en desarrollo desde la década de los años ochenta del siglo XX, hasta el actual momento histórico, son referentes contundentes de la imperiosa exigencia de aportar en la enseñanza de la geografía los fundamentos teóricos y metodológicos que sustentan la perspectiva interdisciplinaria, como base esencial en la gestión por promover su innovación en su práctica escolar cotidiana. Se trata de orientar la acción pedagógica y didáctica desde una opción facilitadora del análisis explicativo e interpretativo más coherente con la realidad estudiada.

Los cambios paradigmáticos y epistemológicos se erigen como orientación de fundamental importancia para proponer el viraje que reiteradamente se ha solicitado desde los años cincuenta del siglo xx, hasta el presente, en la enseñanza geográfica. De allí la exigencia de contribuir con planteamientos para contribuir con iniciativas que mejoren la calidad formativa de los ciudadanos, como actores protagonistas en el mundo globalizado, de la crisis cotidiana, la complejidad y la incertidumbre. 


\section{Consideraciones finales}

Desde fines del siglo XX e inicios del nuevo milenio, se ha hecho frecuente considerar la apremiante exigencia de innovar la enseñanza geográfica en correspondencia con los sorpresivos, inesperados e inciertos acontecimientos de acentuadas dificultades para la sociedad contemporánea. Una opción científica, pedagógica y didáctica propuesta reiterada en los espacios académicos, lo constituye la interdisciplinariedad. Se trata de indagar los estudios desde una visión integral, exhaustiva y penétrate en procura de las razones explicativas, como de la evolución y realidad actual del objeto de estudio. .

En el ánimo de apoyar esta iniciativa por pensar que es una excelente oportunidad para avanzar con el salto epistémico descriptivo hacia el análisis interpretativo fundado en una labor formativa más orientada a atender la necesidades de la sociedad y su transformación. Asimismo, se trata de una iniciativa para contrarrestar la permanencia de una enseñanza aferrada a lo tradicional, debido a su acento pragmático tan enraizado, pero también crónico y alarmante. Por tanto, se hace imprescindible asumir, entre otras las siguientes consideraciones:

a) En el proceso formativo coherente con la realidad del nuevo milenio, se torna ineludible por no decir obligatorio, prestar atención a los sucesos cotidianos a los que tiene acceso la colectividad planetaria, en su vivencia y en los medios de comunicación social. Por tanto, es apremiante mermar la indiferencia de espectadores neutrales, apolíticos y desideologizados, pues de una u otra forma, están notablemente afectados por las implicaciones y repercusiones derivadas de la ocurrencia de los accidentes socioambientales de frecuencia inusitada. En consecuencia, la humanidad vive un escenario inhumano, inmoral y perverso pues a pesar de los aportes contundentes en los diversos ámbitos del quehacer social, es inocultable la ignorancia y el oscurantismo.

b) Ante las condiciones del mundo contemporáneo se impone educar en forma razonada y critica a los ciudadanos, de tal manera que entiendan el mundo vivido y asuman posturas interpretativas sobre el suceder de los acontecimientos actuales. Es necesario comprender el escenario donde las personas no son simples espectadores, sino también actores de los eventos desenvueltos en la cotidianidad de los lugares. En efecto, una alfabetización donde la reflexión debe ser tarea esencial en el fomento de la crítica constructiva y democrática.

c) En el viraje educativo se torna imprescindible comprender que existen otras opciones para elaborar el conocimiento además de la ciencia positiva, pues no es la única acción epistemológica. Por tanto, es el problema quien determina la direccionalidad científica de la investigación a realizar. Se trata de la apertura al conocer al encontrar la posibilidad de asumir los objetos de estudio en estrecha relación con su propia naturaleza. Así, es posible estimar la realidad desde la visión ajustada a su desenvolvimiento real.

d) Entender la innovación implica estimar la conveniencia de dar el salto desde la disciplina hacia lo interdisciplinario. A pesar de la condición de la geografía como ciencia, integral, es beneficioso que aproveche los fundamentos teóricos y metodológicos de las disciplinas afines en el tratamiento de los problemas ambientales, geográficos y sociales. Eso representa para su enseñanza, acudir a ofrecer pedagógica y didácticamente oportunidades desde la versión interdisciplinaria.

e) La enseñanza de la geografía debe evitar su acento pragmático al facilitar, como tradicionalmente lo ha hecho, los procesos de enseñanza y de aprendizaje para el 
simple acto de transmitir datos nocionales y conceptuales ajenos a los acontecimientos del ámbito inmediato. Esta preocupación es reiterativa desde mediados del siglo $\mathrm{XX}$, cuando se iniciaron las reformas curriculares para innovar el acto educante de la geografía. De allí que se pretenda valorizar la reiterada denuncia de su atraso y obsolescencia.

Pretender el uso didáctica de la interdisciplinariedad amerita de iniciativas promotoras de orientaciones educativas, pedagógicas, didácticas y geográficas que cambien el característico rumbo pragmático. Es revisar para mejorar la actividad formativa impregnada de empirismo, indiferencia y desgano pues se erige como un significativo obstáculo epistémico para comprender la difícil realidad del mundo globalizado. De allí la repetición constate en los espacios académicos de su innovación; en especial, en el acto educante en su labor habitual, como objeto de explicación de la emergente episteme cualitativa.

Se reclama entonces una enseñanza geográfica acorde con la transformación de las necesidades más apremiantes de los ciudadanos. Eso representa reorientar la acción formativa con el apoyo de la investigación centrada en la participación y el protagonismo del educando en la explicación de su situación comunitaria. Se impone entonces promover la indagación donde se ejercite el análisis crítico y constructivo con capacidad de diseñar opciones factibles del cambio social forjadoras de la conciencia crítica.

\section{Bibliografía}

Aponte, E. 2006. la geohistoria, un enfoque para el estudio del espacio venezolano desde una perspectiva interdisciplinaria. Scripta Nova. Revista Electrónica de Geografía y Ciencias Sociales. Vol. X, No 218 (08), 1 de agosto de 2006.

Araya P, Fabián R. 2004. Educación geográfica para la sustentabilidad (2005-2014). Revista Quaderns Digital No 37, p. 4-13.

Estefanía, J. 1996. La nueva economía. La Globalización. Madrid: Editorial Debate, S.A.

Ferrer, A. 1996. Historia de la Globalización. Orígenes del orden económico mundial. 2da Edición. Buenos Aires: Fondo de Cultura Económica., S.A.

García Ballesteros, A. 1986. El uso del espacio en la vida cotidiana. Actas de las IV Jornadas de Investigación Interdisciplinaria. Madrid: Universidad Autónoma de Madrid, 9-27.

Grasa, R. 1996. Los nuevos retos de la posguerra fría. Cuadernos de Pedagogía Na 249, 10-15.

Gurevich, R. 1994. Un desafío para la geografía: Explicar el mundo real. Didáctica de las Ciencias Sociales. Buenos Aires: Editorial Paidós, S.A.

Gurevich, R. 2005. Sociedades y territorios en tiempos contemporáneos. Una introducción a la enseñanza de la Geografía. FCE, Buenos Aires.

Hernández Arvelo, M. A. 1998. Transformaciones políticas globales. Globalización económica y regionalización. Globalización y regionalización. Su impacto en las soberanías nacionales. Mérida: Universidad de Los Andes

Hollman, V. C. 2008. La globalización en la geografía escolar: Continuidades y rupturas en la construcción geográfica de un contenido. Biblio 3W Revista Bibliográfica de Geografía y Ciencias Sociales. Universidad de Barcelona Vol. XIII, No 803, 25 de noviembre de 2008.

Kay, J. 2001, enero 23. La geografía y la historia importan a la economía. Diario El Universal, Caracas, Venezuela, p. 2-6).

Lanz, R. 1998. Temas posmodernos. Crítica de la razón pura. Caracas: Fondo Editorial Tropykos. 
Martínez, M. 1989. Comportamiento Humano. Nuevos métodos de investigación. México. Editorial Trillas, S.A.

Martínez, M. 2004. Ciencia y arte en la metodología cualitativa. México: Editorial Trillas, S.A.

McLuhan, H. M. 1974. Teoría de la imagen. Barcelona (España): Salvat Editores, S.A.

Moss, J. 1967. La revolución científica. Madrid. Editorial Ciencia Nueva, S.L.

Naisbitt, J. y Aburdene, P. 1991. Megatendencias 2000. Diez nuevos rumbos para los años noventa. Tercera Edición. Bogotá. Grupo Editorial Norma.

Naisbitt, J. 1984. Megatendencias. Diez nuevas orientaciones que están transformando nuestras vidas. Bogotá. Editorial Printer Colombiana Ltda.

Núñez Tenorio, José Rafael. 1976. Introducción a la ciencia. 7ma Edición. Caracas: Vadell Hermanos Editores.

Ríos Cabrera, P. 2004. La aventura de aprender. 4ta Edición. Caracas: Editorial Cognitus, C. A.

Rodríguez E, Luz A. 2008. Vínculo entre la investigación-acción, el constructivismo y la didáctica crítica. Odiseo Revista Electrónica de Pedagogía. Año 5, No 10. Disponible en: http://www. odiseo.com.mx/2008/5-10/rodriguez-vinculo.html

Santaella, R. 2005. Geografía, diálogo entre sociedad e historia. Caracas, Universidad Central de Venezuela.

Santarelli S. y, Campos, Marta. 2002. Corrientes epistemológicas, metodología y prácticas en Geografía. Universidad Nacional del Sur. EdiUNS.

Tovar López, R. A. 1983. Educación y el equilibrio del sistema sociedad-naturaleza. Revista Geodidáctica Nª1, 9-19.

Villanueva Zarazaga, J. 2002. Algunos rasgos de la geografía actual. Biblio 3W. Revista Bibliográfica de geografía y Ciencias Sociales. Universidad de Barcelona Vol. VII, 342. Disponible en http://www.ub.es/geocrit/b3w-342.htm [ISSN 1138-9796]. 